\title{
Magnetoencephalogram background activity analysis in Alzheimer's disease patients using auto mutual information
}

\author{
Carlos Gómez, Roberto Hornero, Member, IEEE, Alberto Fernández, Daniel Abásolo, Member, IEEE, \\ Javier Escudero*, and Miguel López, Member, IEEE
}

\begin{abstract}
The goal of this study was to analyze the magnetoencephalogram (MEG) background activity in patients with Alzheimer's disease (AD) using the auto mutual information (AMI). Applied to time series, AMI provides a measure of future points predictability from past points. Five minutes of recording were acquired with a 148-channel wholehead magnetometer (MAGNES 2500 WH, 4D Neuroimaging) in 12 patients with probable $A D$ and 12 elderly control subjects. Artifact-free epochs of 20 seconds (3392 points, sample frequency of $169.6 \mathrm{~Hz}$ ) were selected for our study. Our results showed that the absolute values of the averaged decline rate of AMI were lower in AD patients than in control subjects for all channels. In addition, there were statistically significant differences $(p<0.01$, Student's $t$-test) in most channels. These preliminary results suggest that neuronal dysfunction in $A D$ is associated with differences in the dynamical processes underlying the MEG recording.
\end{abstract}

\section{INTRODUCTION}

A LZHEIMER'S disease (AD) is the most common form of dementia [1], a group of conditions that gradually destroys brain cells and leads to progressive decline in mental function. This irreversible brain disorder is characterized by neuronal loss and the appearance of neuritic plaques containing amyloid- $\beta$-peptide and neurofibrillary tangles [2]. As a definite diagnosis of AD is only possible by necropsy, a differential diagnosis with other types of dementia and with major depression is used. It can include Mini-Mental Statues Examination (MMSE) of Folstein [3], magnetic resonance imaging, computerized axial tomography, positron emission tomography, verbal tests, etc.

There are several studies of the electroencephalogram (EEG) and the magnetoencephalogram (MEG) in AD patients with non-linear methods. The most widely used are the correlation dimension $\left(D_{2}\right)$ and the first Lyapunov exponent $\left(L_{1}\right) . D_{2}$ is a measure of the system dimensional complexity $[4,5]$ while $L_{1}$ is a dynamic complexity measure

Manuscript received March 30, 2006. This work has been supported by a project from the Consejería de Fomento de la Junta de Castilla y León. Asterisk indicates corresponding author.

C. Gómez, R. Hornero, D. Abásolo and M. López are with the E. T. S. Ingenieros de Telecomunicación, Universidad of Valladolid, Camino del Cementerio s/n, 47011-Valladolid, Spain (phone: +34 983 423000, ext. 5589; fax: +34 983 423667; e-mails: \{carlos.gomez, roberto.hornero, daniel.abasolo, miguel.lopez\}@tel.uva.es, jescrod@ribera.tel.uva.es).

A. Fernández is with the Centro de Magnetoencefalografía Dr. PérezModrego, Universidad Complutense de Madrid, Spain (e-mail: aferlucas@med.ucm.es). that describes the divergence of trajectories starting at nearby initial states [6]. Jeong et al. [7] showed that AD patients' EEGs exhibit significantly lower $D_{2}$ and $L_{1}$ than the EEGs of control subjects in many channels. Nevertheless, there are several drawbacks in using these measures. Reliable estimations of $D_{2}$ and $L_{1}$ require a large quantity of data [8], and stationary and noise free time series [7]. These assumptions cannot be achieved for physiological data. Moreover, the computational cost is high, especially for great amount of data. Therefore, new non-linear methods are necessary to study the MEG background activity.

Lempel-Ziv complexity measures the complexity of finite sequences and is related to the number of distinct substrings and the rate of their occurrence along the sequence [9]. Recent studies have applied this non-linear method to EEG [10] and MEG signals [11] of $\mathrm{AD}$ patients, finding significant differences in certain regions of the brain. Other method is the approximate entropy, a family of statistics that provides a measure of the complexity and irregularity of a signal [12]. Applied to EEG, Abásolo et al. [13] suggested that the $\mathrm{AD}$ patients' recordings are more regular than the control subjects' ones.

In this study, other non-linear method, the auto mutual information (AMI), has been used to analyze the MEG signals. Mutual information (MI) has been used to study different types of diseases and brain states. Xu et al. [14] computed the complexity of cross mutual information (CMI) functions among $8 \mathrm{EEG}$ channels for four different functional states: awake with opened and closed eyes, light sleep and deep sleep. Na et al. [15] estimated the decreased rate of $\mathrm{AMI}$ and the $\mathrm{CMI}$ values from the EEG of ten schizophrenic patients and ten normal controls.

Both non-linear methods were also applied to study the EEG activity in AD [16]. Jeong et al. found that AMI profiles decreased more slowly with time delay throughout the brain in $\mathrm{AD}$ patients than in control subjects. The CMI analyses showed a significantly decreased transmission of information between pairs of $\mathrm{AD}$ patients' electrodes. Moreover, it has been shown that MI might be useful to predict the response to anesthesia [17].

In this preliminary study, we have examined the MEG background activity in patients with probable $\mathrm{AD}$ and in age-matched control subjects using auto mutual information (AMI). Our purpose is to test the hypothesis that an abnormal type of non-linear dynamics is associated with AD. 
TABLE I

SOCIODEMOGRAPHIC DATA OF AD PATIENTS AND CONTROL SUBJECTS

\begin{tabular}{|c|c|c|c|c|c|c|c|}
\hline Identification & Age & Sex & MMSE & Identification & Age & Sex & MMSE \\
\hline$\overline{\mathrm{Alz}-1}$ & 71 & Female & 15 & Con-1 & 84 & Male & 29 \\
\hline Alz-2 & 83 & Male & 10 & Con-2 & 61 & Female & 29 \\
\hline Alz-3 & 56 & Female & 14 & Con-3 & 70 & Female & 30 \\
\hline Alz-4 & 64 & Female & 15 & Con-4 & 64 & Female & 30 \\
\hline Alz-5 & 59 & Female & 20 & Con-5 & 60 & Male & 30 \\
\hline Alz-6 & 60 & Male & 16 & Con-6 & 63 & Female & 30 \\
\hline Alz-7 & 72 & Female & 15 & Con-7 & 73 & Male & 29 \\
\hline $\mathrm{Alz}-8$ & 71 & Female & 15 & Con-8 & 69 & Female & 29 \\
\hline Alz-9 & 75 & Female & 22 & Con-9 & 56 & Female & 27 \\
\hline Alz-10 & 82 & Female & 21 & Con- 10 & 79 & Male & 29 \\
\hline Alz-11 & 72 & Female & 17 & Con-11 & 79 & Male & 30 \\
\hline Alz-12 & 80 & Male & 24 & Con-12 & 75 & Female & 29 \\
\hline Mean \pm SD & $70.42 \pm 9.04$ & - & $17.00 \pm 3.98$ & Mean \pm SD & $69.42 \pm 8.80$ & - & $29.25 \pm 0.87$ \\
\hline
\end{tabular}

\section{MATERIALS AND METHODS}

\section{A. Subjects}

The MEG data were acquired from 24 subjects. Twelve patients ( 3 men and 9 women) fulfilling the criteria of probable $\mathrm{AD}$ (age $=70.42 \pm 9.04$ years, mean \pm standard deviation SD) have participated in the present study. All of them were recruited from the Asociación de Familiares de Enfermos de Alzheimer (AFAL). The patients were diagnosed according to the criteria of the National Institute of Communicative Disorders and Stroke and the $\mathrm{AD}$ and Related Disorders Association (NINCDS-ADRDA) [18]. The MMSE score was $17.00 \pm 3.98$ (Mean \pm SD). None of the patients used any kind of medication that could have an influence on the MEG.

MEGs were also obtained from 12 age-matched control subjects $(5$ men and 7 women, age $=69.42 \pm 8.80$ years, MMSE $=29.25 \pm 0.87$, mean \pm SD). Sex, age and MMSE score of all subjects are shown in table 1 . The local ethics committee approved the study. All control subjects and all caregivers of the demented patients gave their informed consent for the participation in the current research.

\section{B. $M E G$ recording}

MEG signals were recorded with a 148-channel whole head magnetometer (MAGNES $2500 \mathrm{WH}, 4 \mathrm{D}$ Neuroimaging) in a magnetically shielded room. The subjects lay on a patient bed, in a relaxed state and with their eyes closed. Five minutes of recording were acquired at a sampling frequency of $678.17 \mathrm{~Hz}$. Then, these recordings were down-sampled to $169.549 \mathrm{~Hz}$. Artifact-free epochs of 20 seconds (3292 data points) were selected and filtered with a band-pass filter $(0.5-40 \mathrm{~Hz})$.

\section{Auto mutual information}

Mutual information (MI) provides a measure of both the linear and non-linear statistical dependencies between two time series [16]. It can be defined as the amount of information gained about one signal from the measurement of another [14]. Furthermore, MI between two time series is zero when those series are completely independent, while MI has a maximum value if both series are equal.

We can compute the time-delayed mutual information between $X$ and $Y$ as follows [16]:

$$
\begin{aligned}
I_{X Y_{\tau}}= & \sum_{x(t), y(t+\tau)} P_{X Y_{\tau}}[x(t), y(t+\tau)] \\
& \cdot \log _{2}\left\{\frac{P_{X Y_{\tau}}[x(t), y(t+\tau)]}{P_{X}[x(t)] \cdot P_{Y_{\tau}}[y(t+\tau)]}\right\}
\end{aligned}
$$

where $P_{X}[x(t)]$ and $P_{Y_{\tau}}[y(t+\tau)]$ are the normalized histograms of the distribution of values observed for $x(t)$ and $y(t+\tau)$, while $P_{X Y_{\tau}}[x(t), y(t+\tau)]$ is the joint probability density for the measurements of $x(t)$ and $y(t+\tau)$. The CMI quantifies the information transmitted from one signal to another [16]. Applied to MEG signals, the CMI measures the quantity of information transmitted between certain areas of the brain.

The previous equation can be rewritten as:

$$
\begin{aligned}
I_{X X_{\tau}} & =\sum_{x(t), x(t+\tau)} P_{X X_{\tau}}[x(t), x(t+\tau)] \\
& \cdot \log _{2}\left\{\frac{P_{X X_{\tau}}[x(t), x(t+\tau)]}{P_{X}[x(t)] \cdot P_{X_{\tau}}[y(t+\tau)]}\right\}
\end{aligned}
$$

to calculate the AMI, the MI between $x(t)$ and $x(t+\tau)$. The AMI quantifies the mean predictability of $x(t+\tau)$ from $x(t)$ [16]. To calculate AMI from experimental data is necessary to estimate the joint probability density $P_{X X_{\tau}}$ from histograms. In our study, we have used 8, 16, 32 and 64 bins to construct these histograms.

We evaluated the AMI of all channels over a time delay from 0 to 0.5 seconds. In order to normalize the AMI profiles, they have been divided by the AMI value at $\tau=0$. Hence, AMI values at a zero time delay are always one. The 
slope of the AMI profile was estimated by a line that fits the data in a least-squares sense. This slope was calculated from $\tau=0$ to the first minimum value of the profile. As the decline rate of the AMI is positively correlated with the entropy [19], Jeong et al. [16] suggested that this decrease rate might be used to evaluate the complexity and/or irregularity of a signal.

Finally, a statistical analysis was carried out separately for each channel. Student's $t$-test was used to evaluate the statistical differences between the slopes of the AMI for AD patients and control subjects.

\section{RESULTS}

AMI was estimated for the 148 MEG channels, with a maximum time delay of 0.5 seconds and $8,16,32$ and 64 bins for the construction of the histograms.

Fig. 1 illustrates the normalized AMI profiles of a control subject and an $\mathrm{AD}$ patient at squid A1. This figure shows that the AMI values decrease quickly for low $\tau$ values and then become stable when the values of the time delay $\tau$ increase. As can be noticed, the AMI profile of AD patient declines more slowly than the profile of the control subject. This behavior is the same for all channels. In Fig. 2, absolute values of the decline rate are represented, showing that the AMI profiles decrease more slowly in the AD patients group than in the control group, for all channels. Our results suggest that there are differences between the non-linear
TABLE II

NuMBER OF CHANNELS WITH SIGNIFICANT DIFFERENCES IN THE STUDENT'S TEST

\begin{tabular}{cc}
\hline \hline Histograms & $p<0.01$ \\
\hline 8 bins & 107 channels \\
16 bins & 107 channels \\
32 bins & 98 channels \\
64 bins & 92 channels \\
\hline \hline
\end{tabular}

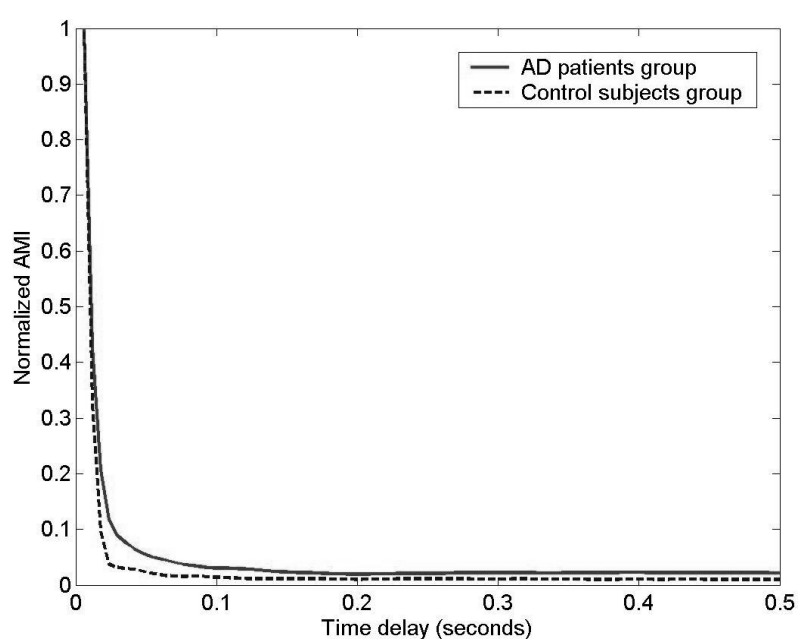

Fig. 1. Normalized AMI curves of the 12 control subjects and the 12 patients with probable AD.
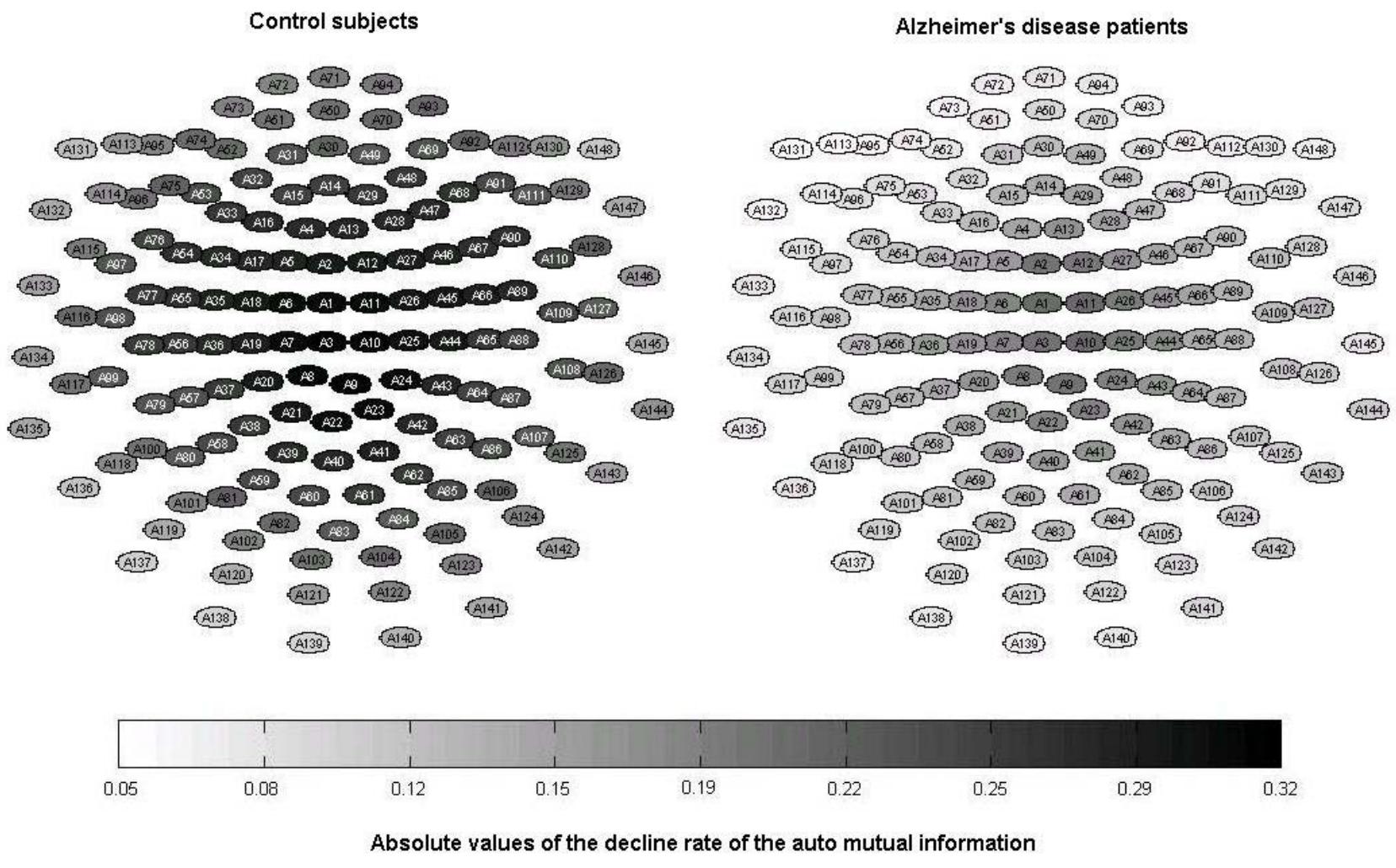

Fig. 2. Absolute values of the averaged AMI decrease rate ( 8 bins for the construction of histograms) for the AD patients and the control subjects in all channels (from A1 to A148). 
dynamics of AD patients and control subjects' MEGs. These differences are statistically significant ( $p<0.01$, Student's $t$ test) in 107 channels for histograms of 8 and 16 bins, in 98 channels for 32 bins and in 92 channels if the histograms are constructed with 64 bins. These results are summarized in Table II.

\section{DisCUSSION AND CONCLUSIONS}

This study presents the AMI as a method to analyze the MEG background activity in 12 patients with probable AD and in 12 control subjects. AMI estimates the degree to which $x(t+\tau)$ can be predicted from $x(t)$. Moreover, the rate of decrease of the AMI with increasing $\tau$ is a normalized complexity measure of the time series [16]. For our pilot study, we have used values of $\tau_{\max }=0.5$ seconds, and 8,16 , 32 and 64 bins to construct the histograms. Furthermore, AMI does not require a large number of data points to be reliably estimated and can be applied to non-stationary time series [16]. Thus, this measure is much better suited for MEG complexity analysis than traditional non-linear techniques as $L_{1}$ or $D_{2}$.

We have found that the absolute values of the average AMI decrease rate are lower in AD patients in all channels. The differences were statistically significant $(p<0.01)$ in 107, 98, 98 and 92 channels for histograms of 8, 16, 32 and 64 bins, respectively. Because AD patients' group and control subjects' group were carefully matched for age, the significantly reduced AMI decline rate may well represent the cognitive dysfunction in $\mathrm{AD}$. However, changes in EEG/MEG activity also appear in other pathological states as schizophrenia [15], vascular dementia [20] and epilepsy [21].

Our results agree with previous studies of the EEG/MEG background activity in AD patients [7], [10], [11], [13]. These studies suggested a loss of complexity and/or irregularity in the brain electrical and magnetic activity in the patients with probable AD. This reduction of complexity on the EEG/MEG background activity in AD might arise from neuronal death, deficiency of neurotransmitters like acetylcholine, and/or loss of connectivity of local neuronal networks [6].

In summary, we have shown that the AMI decrease rate could be a good method to differentiate between AD patients and elderly control subjects. Nevertheless, this is a preliminary study and a larger database is needed to confirm our results.

\section{REFERENCES}

[1] T. D. Bird, "Harrison's principles of internal medicine." in Alzheimer's disease and other primary dementias, E. Braunwald, A. S. Fauci, D. L. Kasper, S. L. Hauser, D. L. Longo, and J. L. Jameson, Eds. New York: The McGraw-Hill Companies Inc, 2001, pp. 23912399.

[2] B. J. Cummings, C. J. Pike, R. Shankle, and C. W. Cotman, "Betaamyloid deposition and other measures of neuropathology predict cognitive status in Alzheimer's disease." Neurobiol. Aging, vol. 17, pp. 921-933, 1996.
[3] M. F. Folstein, S. E. Folstein, and P. R. McHugh, "Mini-mental state: a practical method for grading the cognitive state of patients for the clinician," J. Psychiatr. Res., vol. 12, pp. 189-198, 1975.

[4] P. Grassberger and I. Procaccia, "Characterization of strange attractors," Pyis. Rev. Lett., vol. 50, no. 5, pp. 346-349, Jan. 1983.

[5] P. Grassberger and I. Procaccia, "Estimation of the Kolmogorov entropy from a chaotic signal," Phys. Rev. A, vol. 28, no. 4, pp. 25912593, Oct. 1983.

[6] J. Jeong, "EEG dynamics in patients with Alzheimer's disease," Clin. Neurophysiol., vol. 115, pp. 1490-1505, 2004.

[7] J. Jeong, S. Y. Kim, and S.-H. Han, "Non-linear dynamical analysis of the EEG in Alzheimer's disease with optimal embedding dimension," Electroen. Clin. Neuro., vol. 106, pp. 220-228, 1998.

[8] J. P. Eckmann and D. Ruelle, "Fundamental limitations for estimating dimensions and Lyapunov exponents in dynamical systems," Physica $D$, vol. 56, pp. 185-187, 1992.

[9] A. Lempel and J. Ziv, "On the complexity of finite sequences," IEEE Trans. Inform. Theory, vol. 22, pp. 75-81, 1976.

[10] D. Abásolo, R. Hornero, C. Gómez, M. García, and M. López, "Analysis of EEG background activity in Alzheimer's disease patients with Lempel-Ziv complexity and Central Tendency Measure," Med. Eng. Phys., vol. 28, pp. 315-322, 2006.

[11] C. Gómez, R. Hornero, D. Abásolo, A. Fernández, and M. López, "Complexity analysis of the magnetoencephalogram background activity in Alzheimer's disease patients," Med. Eng. Phys., to be published.

[12] S. M. Pincus, "Approximate entropy as a measure of system complexity," in Proc. Natl. Acad. Sci. USA, vol. 88, Mar. 1991, pp. 2297-2301.

[13] D. Abásolo, R. Hornero, P. Espino, J. Poza, C. I. Sánchez, and R. de la Rosa, "Analysis of regularity in the EEG background activity of Alzheimer's disease patients with approximate entropy," Clin. Neurophysiol., vol. 116, no. 8, pp. 1826-1834, Aug. 2005.

[14] J. Xu, Z. -R. Liu, R. Liu, and Q. -F. Yang, "Information transmission in human cerebral cortex," Physica D, vol. 106, pp. 363-374, 1997.

[15] S. H. Na, S.-H. Jin, S. Y. Kim, and B.-J. Ham. "EEG in schizophrenic patients: mutual information analysis," Clin. Neurophysiol., vol. 113, pp. 1954-1960, 2002.

[16] J. Jeong, J. C. Gore, and B. S. Peterson. "Mutual information analysis of the EEG in patients with Alzheimer's disease," Clin. Neurophysiol., vol. 112, pp. 827-835, 2001.

[17] L. Huang, P. Yu, F. Ju, and J. Cheng. "Prediction of response to incision using the mutual information of electroencephalogram during anaesthesia," Med. Eng. Phys., vol. 25, pp. 321-327, 2003.

[18] G. McKhann, D. Drachman, and M. Folstein, "Clinical diagnosis of Alzheimer's disease: report of NINCDS-ADRDA work group under the auspices of department of health and human services task force on Alzheimer's disease," Neurology, vol. 34, pp. 939-944, 1984.

[19] M. Palus, "Coarse-grained entropy rates for characterization of complex time series," Physica D, vol. 93, pp. 64-77, 1993.

[20] J. Jeong, J. H. Chae, S. Y. Kim, and S. H. Han, "Nonlinear dynamic analysis of the EEG in patients with Alzheimer's disease and vascular dementia," J. Clin. Neurophysiol., vol. 18, pp.58-67, 2001.

[21] H. Jing and M. Takigawa, "Comparison of human ictal, interictal and normal non-linear component analices," Clin. Neurophysiol., vol. 111, pp. 1282-1292, 2001. 